\title{
Hemangioma cavernoso multifocal en cráneo. Revisión de un caso
}

\author{
A. Vega; E. De Obieta; G. Aguado; M. Esqueda; S. Ruíz; E. Ramirez y J. Pérez*
}

Servicios de Neurocirugía y Neuro-Patología*. Hospital General Naval de Alta Especialidad. Coyoacán. México.

\section{Resumen}

Presentamos el caso clínico de una paciente de 39 años de edad quien presenta dos tumoraciones en cráneo a nivel frontal derecho y parietal izquierdo, que fueron resecadas en bloque mediante craniectomías guiadas por navegación. El defecto óseo fue reconstruido con mallas de titanio. El reporte histopatológico fue de hemangioma óseo en ambas lesiones. El seguimiento a 6 meses posterior a la cirugía sin evidencia de recurrencia y con un resultado cosmético satisfactorio.

PALABRAS CLAVE: Hemangioma intraóseo. Tumores del cráneo. Hemangiomas craneales múltiples.

Multifocal cavernous hemangioma of the skull. Case report

\section{Summary}

A 39 year old female presented with a multifocal lesions in the skull, at the frontal right and parietal left. We performed bilateral craniectomies guided with navigation, and the bone defects were repaired with titanium mesh. The pathological examination reported intraosseous cavernous hemangioma in both lesions. Follow up of six months without any complication or recurrence and good cosmetic outcome.

KEY WORDS: Intraosseous hemangioma. Skull tumors. Multiple cavernous hemangioma.

\section{Introducción}

Los hemangiomas intraóseos son tumores benignos de origen vascular. Este tipo de neoplasias son poco comunes y representan el $0.7 \%$ dentro de los tumores óseos y de éstos solo el $0.2 \%$ involucran los huesos del cráneo,

Recibido: 1-06-10. Aceptado: 20-09-10 siendo el hueso parietal y frontal los sitios principalmente afectados $^{6}$. La primera descripción de un hemangioma en los huesos del cráneo fue descrita en 1845 por Toynbee ${ }^{22}$. Este tipo de lesiones tienen predilección por el género femenino (3:1) y se diagnostican más frecuentemente dentro de la cuarta y quinta década de la vida. La presentación multifocal es una condición poco común ya que en la mayoría de los casos debutan como lesiones únicas ${ }^{2}$.

Presentamos el caso de una paciente con hemangiomas múltiples en cráneo, la cual fue tratada mediante la resección en bloque de las lesiones guiado por neuronavegación y la reconstrucción con malla de titanio.

\section{Caso clínico}

Se trata de paciente del sexo femenino de 39 años de edad sin antecedentes de importancia. Presenta aumento de volumen en región frontal derecha de un año de evolución. A la exploración física se palpa una tumoración indurada en región frontal derecha que mide $3 \times 3 \mathrm{~cm}$. La exploración física neurológica no revela anormalidades. En el estudio de tomografía de cráneo se observa una lesión expansiva ósea a nivel frontal derecho y otra a nivel parietal izquierdo, que se confirman en los estudios de reconstrucción 3D (Figura 1). En los estudios de resonancia magnética se observan lesiones hipointensas en T1 e hiperintensas en T2 que refuerzan con aplicación de gadolinio, con ausencias de señal a nivel intralesional, que condicionan compresión del lóbulo frontal derecho (Figura 2). La tomografía por emisión de positrones presenta lesiones hipermetabólicas óseas a nivel de cráneo sin evidencia de actividad metabólica a otro nivel.

Se le realizó resección en bloque de ambas lesiones mediante una craniectomía frontal derecha y parietal izquierda guiada por neuronavegación (Figura 3) para obtener bordes quirúrgicos libres, y se llevo a cabo una reconstrucción de los defectos craneales con malla de titanio (Figura 4).

El reporte histopatológico fue de un hemangioma óseo de variedad cavernoso en ambas lesiones (Figura 5).

La paciente evolucionó satisfactoriamente en el poso- 


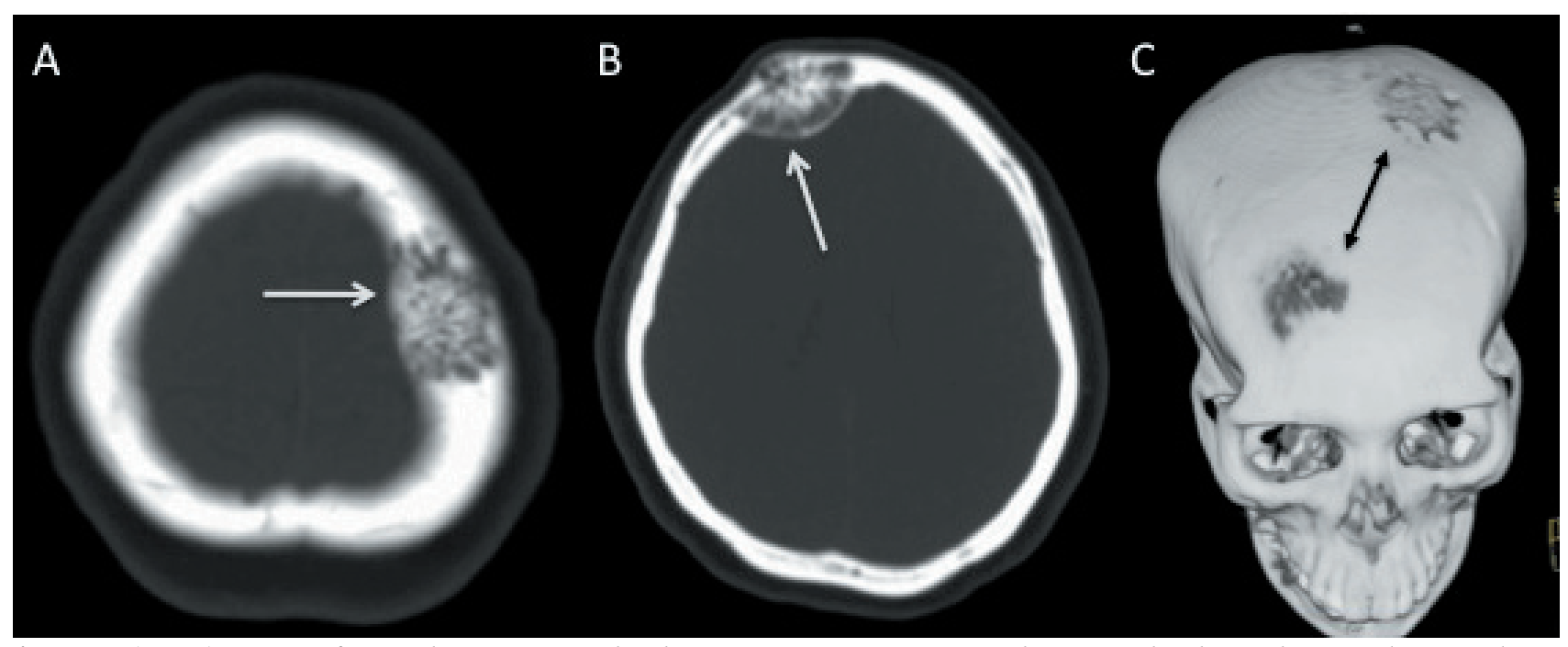

Figura 1. (A y B)Tomografía axial computarizada de cráneo con ventana para hueso en donde se observan lesiones líticas óseas en panal de abejas a nivel parietal izquierdo (A) y frontal derecho (B) . (C) Reconstrucción $3 D$ del mismo estudio en donde se evidencian ambas lesiones.

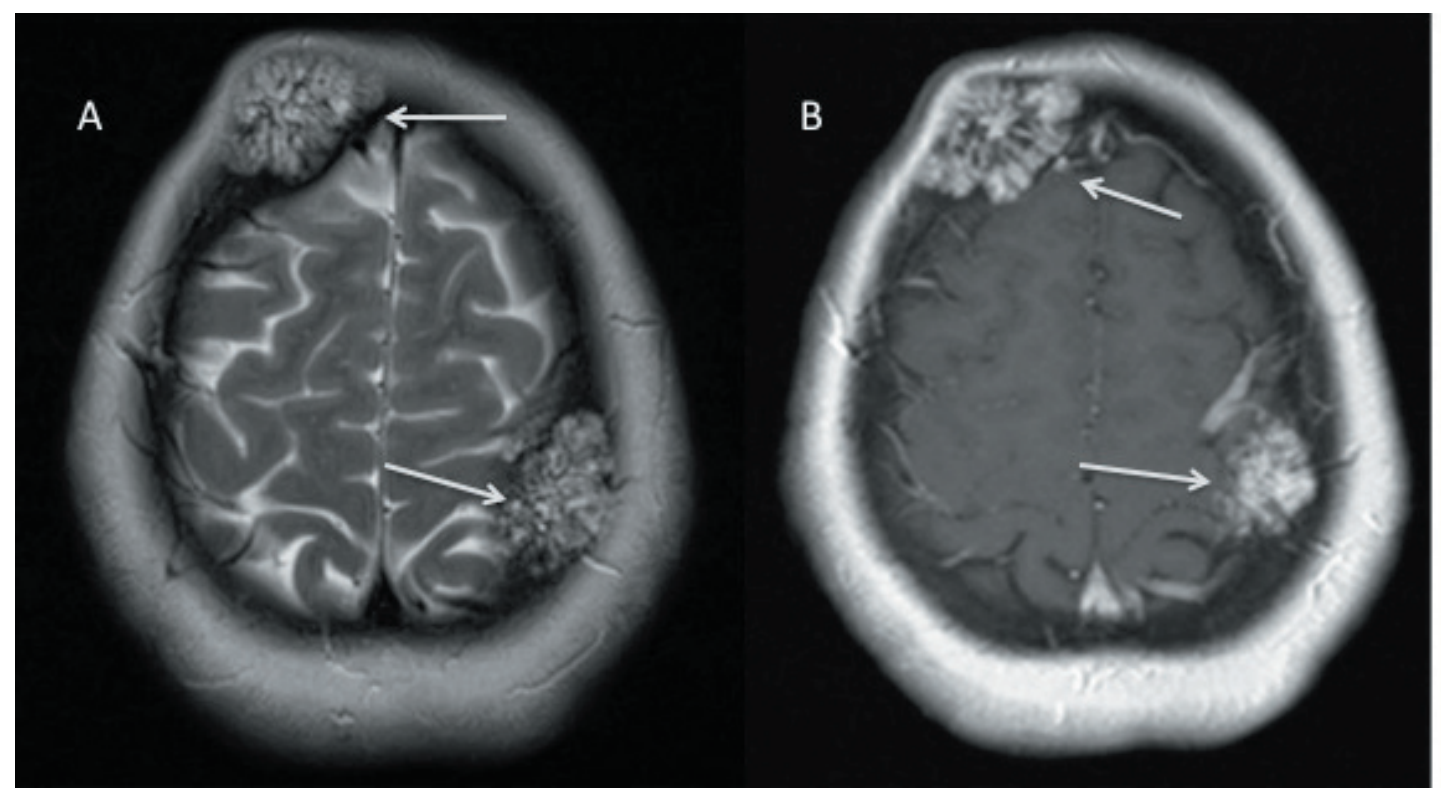

Figura 2. (A) Secuencia T2 de la resonancia magnética de cráneo apreciándose ambas lesiones (flechas) hiperintensas heterogéneas con ausencias de señal. (B) Secuencia T1 con gadolinio en donde se aprecian ambas lesiones de las mismas características hiperintensas que refuerzan a la administración del gadolinio.

peratorio, fue egresada al quinto día posterior a la cirugía. En el seguimiento a seis meses se encuentra sin evidencia de recurrencia tumoral y con un resultado cosmético satisfactorio.

\section{Discusión}

Los hemangiomas del esqueleto son tumores de crecimiento muy lento y representan sólo el $0.7 \%$ de todas las neoplasias óseas y la mayoría se presentan en la columna vertebral. Los hemangiomas intraóseos de la calota son raros, usualmente son del tipo cavernoso y representan sólo el $0.2 \%$ de todas las neoplasias óseas ${ }^{4,13}$. Sin embargo, constituyen el segundo tumor primario más común del cráneo después del osteoma y en múltiples series comprende hasta el $10 \%$ de todas las neoplasias benignas del cráneo $^{5}$. Estos tumores son 2 ó 3 veces más frecuentes en mujeres que en hombres; se pueden encontrar en pacientes de todas las edades pero se diagnostican más frecuentemente en las décadas medias de la vida, con un pico de inci- 


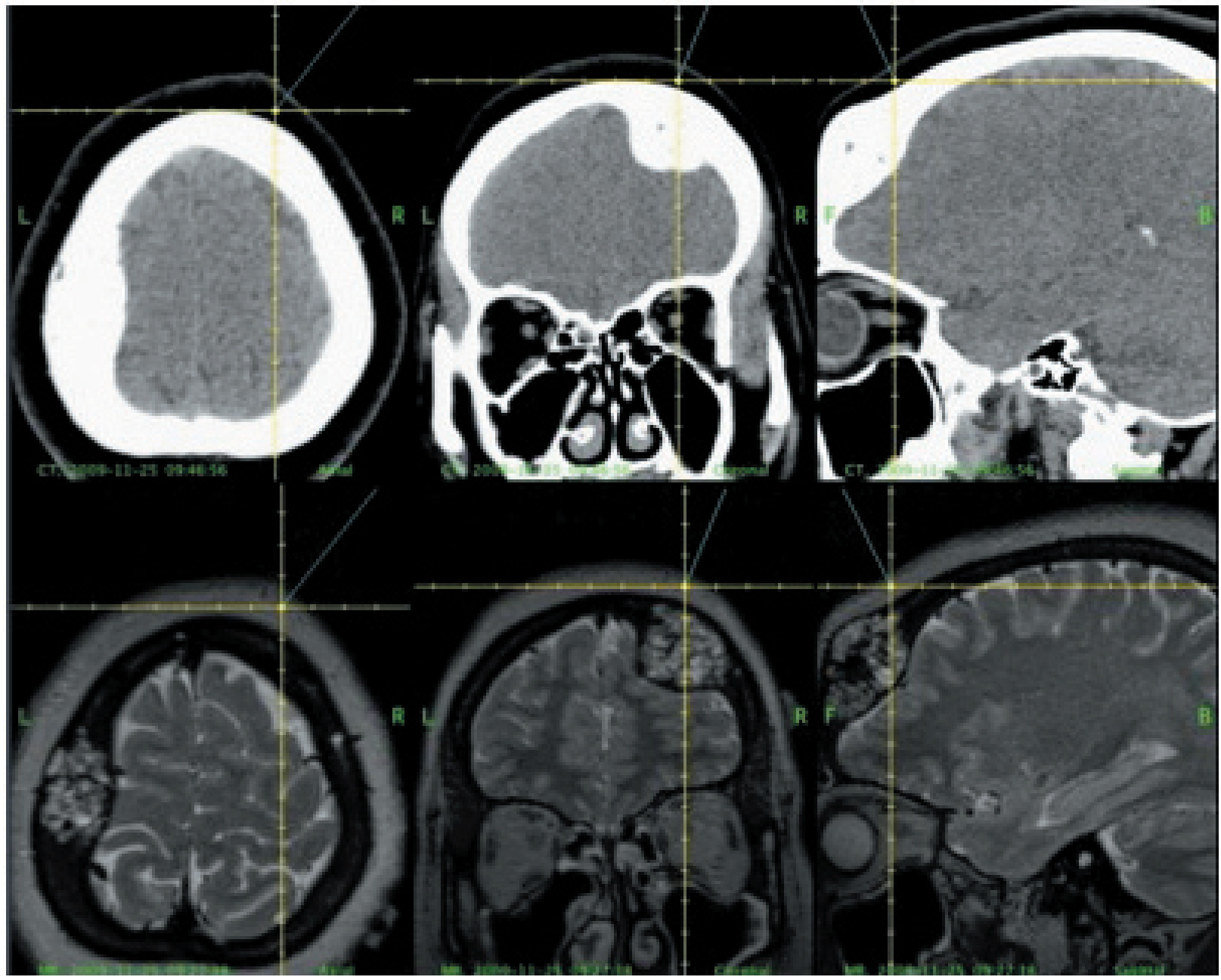

Figura 3. Neuronavegación intraoperatoria con imágenes de tomografia y resonanacia magnética para definir bordes de craniectomia.

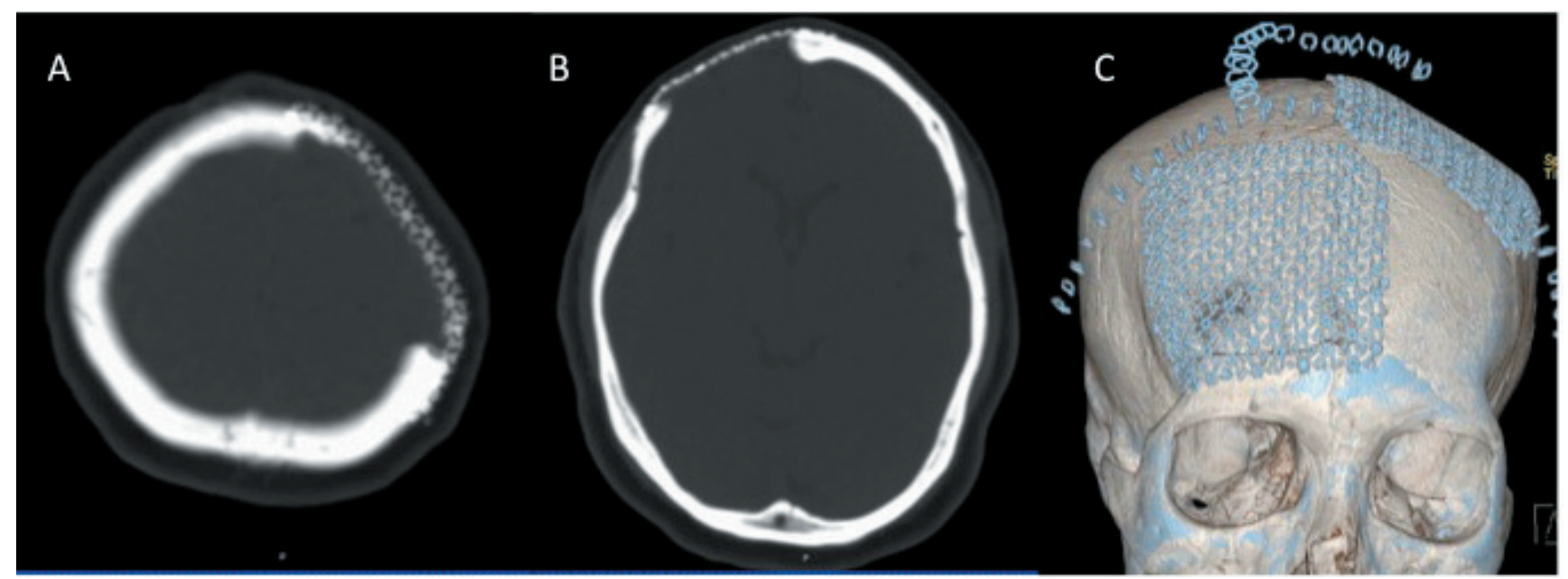

Figura 4. (A y B) Tomografía de cráneo simple con ventana ósea en la cuál se observa la craneoplastía con malla de titanio. (C) Tomografía de cráneo con reconstrucción $3 D$ de ambas craneoplastías. 


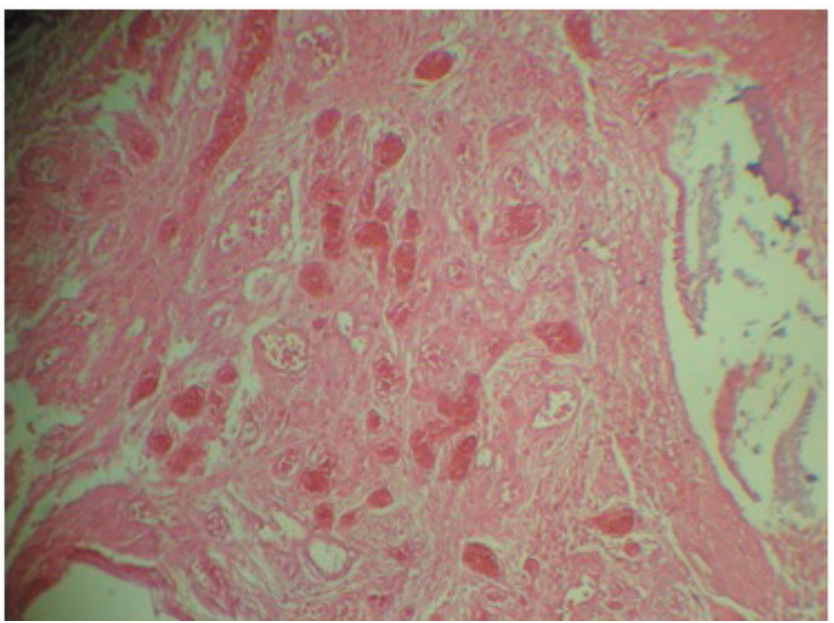

Figura 5. (Hematoxilina-Eosina 40x). Microfotografia de la lesión en la que se observan grandes espacios dilatados, tapizados de endotelio, que contienen elementos formes de la sangre y dichos espacios se encuentran separados por tejido conjuntivo disperso, así como por trabéculas óseas.

dencia en la cuarta década de la vida. Su localización más frecuente en el cráneo son los huesos frontal y parietales, como en el presente caso, y raramente el occipital y temporal, aunque pueden presentarse prácticamente en cualquier hueso del cráneo ${ }^{7}$. Se desconoce la patogenia pero se cree que puede ser congénito o relacionado con algún traumatismo previo ${ }^{14}$. Ciertos autores se inclinan a considerarlos hamartomas de origen mesenquimático más que verdaderas neoplasias vasculares ${ }^{16}$. Existe una forma hereditaria rara de la enfermedad que se encuentra confinada a los huesos craneofaciales ${ }^{12,11}$.

Estas lesiones crecen entre la tabla externa e interna del cráneo y se encuentran irrigadas por ramas de la arteria carótida externa en el espacio diploico. Histológicamente existen 2 tipos de hemangiomas: cavernoso y capilar, el tipo cavernoso se observa en la radiografía simple de cráneo como una lesión redondeada u ovalada de aspecto trabecular, intradiploica, no encapsulada, de márgenes bien definidos, por lo general no esclerosados. Pueden seguir patrones característicos en panal de abejas, rayos de sol en la cortical o estriaciones, que se visualizan fundamentalmente cuando se trata de lesiones de gran tamaño. Generalmente erosionan la tabla externa, manteniendo intacta la interna, no así en esta paciente en quién ambas lesiones destruyen ambas tablas, interna y externa. Estas características son mejor definidas en la tomografía de cráneo, especialmente para lesiones más pequeñas; suele usarse para confirmar la sospecha y ver si existe extensión intracraneal o intradiploica. La resonancia magnética también es una buena técnica diagnóstica, mostrando una lesión hiperintensa en $\mathrm{T} 1$ y $\mathrm{T} 2$ que realza con contraste.
La angiografía no suele ser útil, ya que se trata de lesiones de bajo flujo, aunque puede mostrar típicamente una lesión vascular sin drenaje venoso ${ }^{21}$.

Normalmente son lesiones únicas aunque se han reportado también pacientes con múltiples hemangiomas. En una revisión (Thomas et al. 1975) encontró que sólo 2 de 43 pacientes con hemangiomas tenían múltiples lesiones ${ }^{20}$, sólo se han reportado 9 casos comprobados histológicamente con hemangiomas múltiples en cráneo (Tabla 1) 1,3,7-10,15,19,2. En este caso la paciente presentaba dos lesiones óseas a nivel de hueso frontal derecho y parietal izquierdo, confirmadas por histopatología como hemangiomas cavernosos con lo cual cumple los criterios para ingresar como el caso número diez a los reportados previamente en la literatura.

El diagnóstico diferencial incluye la displasia fibrosa, osteoma, histiocitosis de células de Langerhans, meningioma, enfermedad metastásica, tumor dermoide y mieloma múltiple ${ }^{18}$.

El tratamiento es la resección en bloque de la lesión cuando existe efecto de masa y compromiso neurológico, así como para mejorar la deformidad cosmética y obtener diagnóstico definitivo. Pilcher reportó la primera exéresis exitosa de un hemangioma del cráneo en 1894³ . En 1923 Cushing reporto la resección en bloque del tumor con un borde de hueso normal ${ }^{18}$. El tratamiento quirúrgico de elección es la exéresis en bloque ${ }^{2}$, esta técnica disminuye la pérdida sanguínea transoperatoria aunque esto no sea posible cuando se encuentra involucrada la base del $\mathrm{cráneo}^{17}$. El uso del neuronavegador garantiza la resección de la lesión con bordes libres, aun cuando exista extensión intradiploica no visible durante la cirugía, como lo fue en nuestro caso.

La embolización preoperatoria es una herramienta útil ya que se trata de lesiones que tienen como principal complicación quirúrgica el abundante sangrado transoperatorio principalmente cuando se pretende realizar una craneotomía con curetaje de la lesión y preservación de la tabla externa ${ }^{21}$.

La radioterapia se reserva para aquellos tumores que fueron resecados parcialmente o en caso de recurrencia ${ }^{17}$. El pronóstico después de una escisión completa es excelente y la recurrencia usualmente es rara $^{13}$, sin embargo, los pacientes con hemangiomas craneales múltiples deben ser sometidos a una examinación general ya que pueden presentar hemangiomas en otros órganos ${ }^{10,23,2}$, no siendo el caso para el paciente que presentamos.

\section{Conclusiones}

Los hemangiomas son tumores poco frecuentes de aparición en cráneo y la presencia de lesiones múltiples hacen de esta patología una condición poco frecuente de acuerdo con lo reportado en la literatura. El tratamiento al igual que en los pacientes con lesión única es la resección en bloque 
Tabla1

Casos reportados de hemangiomas multiples en cráneo comprobados histológicamente

\begin{tabular}{|c|c|c|c|c|c|c|}
\hline $\begin{array}{l}\text { No. de } \\
\text { caso }\end{array}$ & Autor (año) & $\begin{array}{l}\text { Edad(años)/ } \\
\text { Género }\end{array}$ & $\begin{array}{l}\text { Localización } \\
\text { de la lesión }\end{array}$ & Tratamiento & Tipo histológico & $\begin{array}{l}\text { Asociación } \\
\text { con otros órganos }\end{array}$ \\
\hline 1 & $\begin{array}{l}\text { Peterson et } \\
\text { al. }(1992)^{15}\end{array}$ & $64 / \mathrm{M}$ & $\mathrm{F}, \mathrm{O}$ & Cirugía & Cavernoso & Ninguna \\
\hline 2 & $\begin{array}{l}\text { Kumar et. } \\
\text { al. }(1996)^{9}\end{array}$ & $45 / F$ & $\mathrm{P}, \mathrm{O}, \mathrm{T}$ & Cirugía & Cavernoso & Ninguna \\
\hline 3 & Corr $(2000)^{3}$ & $28 / F$ & $\mathrm{~F}, \mathrm{O}$ & Biopsia & Cavernoso & Ninguna \\
\hline 4 & $\begin{array}{l}\text { Khanam et. } \\
\text { al. }(2001)^{7}\end{array}$ & $51 / \mathrm{M}$ & $\mathrm{P}, \mathrm{O}$ & Cirugía & Cavernoso & Ninguna \\
\hline 5 & $\begin{array}{l}\text { Kuzeyli et } \\
\text { al. }(2003)^{10}\end{array}$ & $42 / F$ & F, P & Cirugía & Cavernoso & Ninguna \\
\hline 6 & $\begin{array}{l}\text { Koulouris \& } \\
\text { Rao }(2005)^{8}\end{array}$ & 4 días/F & Max, F, C, T, T & Biopsia & Capilar & Piel \\
\hline 7 & $\begin{array}{l}\text { Buhl et al. } \\
(2007)^{1}\end{array}$ & $20 / \mathrm{M}$ & F, O (de novo) & Cirugía & Cavernoso & Ninguna \\
\hline 8 & $\begin{array}{l}\text { Syal et al. } \\
(2007)^{19}\end{array}$ & $20 / \mathrm{M}$ & P, Man & Biopsia, EMB & ND & $\begin{array}{l}\text { Vértebra, } \\
\text { pelvis, tibia }\end{array}$ \\
\hline 9 & $\begin{array}{l}\text { Sasagawa et } \\
\text { al. }(2009)^{18}\end{array}$ & $55 / \mathrm{F}$ & $\mathrm{F}, \mathrm{T}, \mathrm{P}, \mathrm{P}$ & Cirugía & Cavernoso & Hígado \\
\hline 10 & Caso presente & 39/M & $\mathrm{F}, \mathrm{P}$ & Cirugía & Cavernoso & Ninguna \\
\hline
\end{tabular}

F: hueso frontal, Man: mandíbula, Max: maxila, ND: no descrito, O: hueso occipital, P: hueso parietal, T: hueso temporal, EMB: embolización, C: cigoma

de la lesión y en el caso de lesiones multifocales en cráneo se deben realizar los estudios de extensión para descartar hemangiomas en otros órganos.

\section{Bibliografía}

1. Buhl, R., Barth, H., Döner, L., Nabavi, A., Rohr, A., Mehdorn, HM.: De novo development of intraosseous cavernous hemangioma. J Clin Neurosci 2007; 14: 289-292.

2. Carrasco, R., García, E., Navas, M., Llano, M.A., Sola, R.G.: Hemangioma cavernoso intraóseo craneal. Neurocirugía 2009; 20: 559-562.

3. Corr, P.: Multiple calvarial haemangiomas. Australas Radiol 2000; 44: 118-120.

4. Dogan, S., Kocaeli, H., Sahin, S., Korfali, E., Sarayda- roglu, Ö.: Large cavernous hemangioma of the Frontal Bone: Case report. Neurol Med Chir 2005; 45: 264-267.

5. Goldbrunner, R.: Tumors of the Skull. En Tonn JC (ed). Neuro-Oncology of CNS Tumors. Berlín; Springer, 2006; pp. 76-77.

6. Heckl, S., Aschoff, A., Kunze, S.: Cavernomas of the skull: review of the literature 1975-2000. Neurosurg Rev 2002; 25: 56-67.

7. Khanam, H., Lipper, M.H., Wolff, C.H., Lopes, M.B.S.: Calvarial Hemangiomas: Report of two cases and review of the literature. Surg Neurol 2001; 55: 63-67.

8. Koulouris, G., Rao, P.: Multiple congenital cranial hemangiomas. Skeletal Radiol 2005; 34: 485-489.

9. Kumar, N.A., Ranganadham, P., Bhaskar, G., Chowdhury, A.R.: Multiple calvarial cavernous haemangiomas: case 
report and review of the literature. Neuroradiology 1996; 38: 83-85.

10. Kuzeyli, K, Usul, H, Cakir, E, Caylan, R, Rei, A, Baykal, S, et al.: Multifocal intradiploica cavernous hemngioma of the skull associated with nasal osteoma. Acta Neurochir 2003; 145: 323-326.

11. Leao, B., Cardoso, G.T., Alves, A., Barbosa, W., Castro, R.A.: Primary hemangioma of the skull. Arq Neuropsiquiatr 2008; 66: 569-571.

12. Liu, J.K., Burger, P.C., Harnsberger, H.R., Couldwell, W.T.: Primary Intraosseous Skull Base Cavernous Hemangioma: Case Report. Skull Base 2003; 13: 219-227.

13. Naama, O., Gazzaz, M., Akhaddar, A., Belhachmi, A., Asri, A., Elmostarchid, B., et al.: Cavernous hemangioma of the skull: 3 case reports. Surgical Neurology 2008; 70: 654659 .

14. Nasser, K., Hayashi, N., Kurosaki, K., Hasegawa, S., Kurimoto, M., Mohammed, A., et al.: Intraosseous cavernous hemangioma of the frontal bone: Case report. Neurol Med Chir 2007; 47: 506-508

15. Peterson, D.L., Murk, S.E., Story, J.L.: Multifocal cavernous hemangioma of the skull: report of a case and review of the literature. Neurosurgery 1992; 30: 778-782.

16. Romera, M., Medel, R., Ayala, E., Martin, S., Huguet, P.: Hemangioma intraóseo primario del techo orbitario. Annals d'Oftalmologia 2004; 12: 106-109.

17. Salunke, P., Sinha R., Khandelwal, N.K., Kumar, A., Gupta, K., Mukherjee, K.K.: Primary intraosseus cavernous hemangioma of the skull base. Br J Neurosurg 2010; 24: 8485 .

\section{Comentario al trabajo Hemangioma cavernoso multi- focal en cráneo. Revisión de un caso de A. Vega y cols.}

Vega y colaboradores presentan el caso de una mujer de 39 años con dos cavernomas de la bóveda craneal, una localización infrecuente, como lo es más aún el hecho de que se trate de una lesión multifocal. Es de agradecer el interés de los autores por publicar el caso. Creemos conveniente, sin embargo, introducir algunos comentarios.

Antes de la incorporación de la resonancia magnética en la práctica clínica, existía cierta confusión respecto a la nomenclatura y naturaleza de estas malformaciones vasculares que recibieron múltiples denominaciones como, angiomas cavernosos, hemangiomas cavernosos, malformaciones vasculares angiográficamente ocultas, malformaciones crípticas, malformaciones cavernosas. La localización más habitual de los angiomas cavernosos son
18. Sasagawa, Y., Akai, T., Yamamoto, K., Masuoka, T., Itou, S., Oohashi, M., et al.: Multiple Cavernous Hemangiomas of the Skull Associated With Hepatic Lesions: Case Report. Neurol Med Chir 2009; 49: 162-166.

19. Syal, R., Tyagi, I., Goyal, A., Barai, S., Parihar, A.: Multiple Intraosseous hemangiomas - investigation and role of N-butylcyanoacrylate in management. Head Neck 2007; 29: 512-517.

20. Thomas, J.E., Baker, H.L Jr.: Assessment of roentgenographic lucencies of the skull: a systematic approach. Neurology 1975; 25: 99-106.

21. Torne, L., Martín, J., Ara, J.R.: Hemangioma cavernoso del hueso frontal con hematoma subdural. Neurología 2009; 24:136-142.

22. Toynbee, J.: An account of two vascular tumours developed in the substance of bone. Lancet 1845; 2: 676.

23. Wyke, D.B.: Primary hemangioma of the skull: A rare cranial tumor. Am J Roentgenol 1949; 61: 302-316.

Vega, A.; De Obieta, E.; Aguado, G.; Esqueda, M.; Ruíz, S.; Ramirez, E.; Pérez, J.: Hemangioma cavernoso multifocal en cráneo. Revisión de un caso. Neurocirugía 2010; 21: 484-490.

Correspondencia. Dr. Alfonso Vega. Servicio de Neurocirugía. Hospital General Naval de Alta Especialidad. Eje 2 Ote. Tmo. Heroica Escuela Naval Militar No. 701 Col. Presidentes ejidales. Del. Coyoacán. México DF. CP. 04477.

Email: drvega@neurocirugia.com. los hemisferios cerebrales. ¿Son estas malformaciones vasculares comparables desde el punto de vista biológico a las descritas lesiones craneales? En ese caso, es de interés conocer los antecedentes familiares de la paciente y en su caso, proponer estudios diagnósticos apropiados a la familia, de forma similar a los casos de cavernomas cerebrales múltiples, visto que los cavernomas múltiples suelen ser de origen hereditario ${ }^{2,3}$.

En cuanto a la técnica quirúrgica, no vemos la necesidad de introducir la Neuronavegación en este tipo de lesiones craneales que se palpan a través del cuero cabelludo y que sus márgenes pueden ser establecidos mediante inspección en el momento de la cirugía. Respecto a la posibilidad de embolización preoperatoria sugerida por los autores, sería 
interesante conocer si en el caso que presentan, las lesiones eran sangrantes o no y se hizo algún estudio de imagen vascular preoperatorio.

Finalmente, no creemos que la radioterapia sea una opción a considerar para casos similares al que se presenta, puesto que se trata de una lesión hamartomatosa, es decir no neoplásica, cuya resección quirúrgica completa es curativa, consiguiendo tres objetivos: diagnóstico, terapéutico y cosmético. De acuerdo con los autores y siguiendo al grupo de García de Sola ${ }^{1}$, la radioterapia se debe reservar para aquellas lesiones que se consideran irresecables o en casos de recidiva.

\section{Bibliografía}

1. Carrasco-Moro, R., García-Navarrete, E., Navas-García, M., Andrados de Llano, M., García de Sola R.: Hemangioma cavernoso intraóseo craneal. Neurocirugía 2009; 20: 559-562.

2. Maraire, J.N., Awad, I.A.: Intracranial Cavernous Malformations: Lesion Behavior and Management Strategies. Neurosurgery 1995; 37: 591-605.

3. Washington, C.W., McCoy, K.E., Zipfel, G.J.: Update on the natural history of cavernous malformations and factors predicting aggressive clinical presentation. Neurusurgical Focus 2010; 29: E7.
E. Urculo

N. Samprón

San Sebastián 